\title{
Absence of magnetically induced fractional quantization in atomic contacts
}

\author{
C. Untiedt, ${ }^{1,2}$ D. M. T. Dekker, ${ }^{1}$ D. Djukic, ${ }^{1}$ and J. M. van Ruitenbeek ${ }^{1}$ \\ ${ }^{1}$ Kamerlingh Onnes Laboratorium, Universiteit Leiden, Postbus 9504, NL-2300 RA Leiden, The Netherlands \\ ${ }^{2}$ Departamento de Física Aplicada, Universidad de Alicante, Campus de San Vicente del Raspeig, E-03690 Alicante, Spain
}

(Received 6 October 2003; revised manuscript received 18 November 2003; published 9 February 2004)

\begin{abstract}
Using the mechanically controlled break junction technique at low temperatures and under cryogenic vacuum conditions we have studied atomic contacts of several magnetic ( $\mathrm{Fe}, \mathrm{Co}$, and $\mathrm{Ni}$ ) and nonmagnetic $(\mathrm{Pt})$ metals, which recently were claimed to show fractional conductance quantization. In the case of pure metals we see no quantization of the conductance nor half quantization, even when high magnetic fields are applied. On the other hand, features in the conductance similar to (fractional) quantization are observed when the contact is exposed to gas molecules. Furthermore, the absence of fractional quantization when the contact is bridged by $\mathrm{H}_{2}$ indicates the current is never fully polarized for the metals studied here. Our results are in agreement with recent model calculations.
\end{abstract}

DOI: 10.1103/PhysRevB.69.081401

PACS number(s): 73.63.Rt, 75.75.+a

When a metallic wire is stretched its conductance becomes smaller as a result of the decrease of its cross section. This process continues until the breaking of the wire, and just before this event takes place, the contact is formed by just one atom. In this way atomic-sized contacts between two metallic electrodes can be formed and studied. The instruments that have made these studies possible are the mechanically controllable break junctions and the scanning tunneling microscope. In both techniques the relative displacement of two electrodes is controlled with a resolution of a few picometers by the use of a piezoelectric element which allows us to monitor the formation and breaking of the contact between the two electrodes.

Properties of such atomic-sized contacts have been extensively studied during the past decade $^{1}$ for many different metals both magnetic and nonmagnetic. The conductance of these contacts can be described by the Landauer formula

$$
G=G_{0} \sum_{i} T_{i}
$$

where the summation is extended to all the available channels for the electrons traversing the contact, $T_{i}$ is a number between 0 and 1 for the transmission of the $i$ th channel, and $G_{0}=2 e^{2} / h$ the quantum of conductance (assuming degeneracy of spin) in terms of the electron charge $e$ and Planck's constant $h$. In the case in which the degeneracy of spin would be removed the channels would have to be redefined for each spin and each of these would carry up to $\frac{1}{2} G_{0}$.

The number of channels available in a one-atom contact is determined by the valence of the metal, ${ }^{2}$ and the transmission of each channel is influenced by other parameters such as the number of neighbors or the bond distance. ${ }^{3,4}$ For special cases ( $s$-type metals such as $\mathrm{Au}$ or $\mathrm{Na}$ ), electronic transport through a single atom will be due to a single channel with a transmission close to unity, but this will not be true for other metals where all kinds of combinations of channels with different transmissions will add up to produce the total conductance of the atom. This is the case for transition metals with partial occupation of the $d$ orbitals and therefore they are not expected to have a one-atom conductance of $\frac{1}{2} G_{0}$ or $1 G_{0}$ for magnetic or nonmagnetic metals, respectively. ${ }^{5-7}$ This will make it difficult to establish just from the conductance whether the spin degeneracy of the conductance channels has been lifted or not. However, several claims have appeared of the observation of half-integer conductance quantization for both magnetic and nonmagnetic metals. ${ }^{8-13}$ Some related results have already appeared earlier and have been discussed in Sec. 5.4 of Ref. 1. The claims are based on the observation of peaks in conductance histograms at half integers of the quantum of conductance in experiments made at room temperature. These observations cannot be understood from the present knowledge of transport properties of atomic-sized contacts. Especially the claim of the observation of values of conductance at half integers of the quantum in the case of $\mathrm{Pt}$ and the interpretation of this phenomenon as a result of the lifting of the spin degeneracy ${ }^{13}$ seem to contradict previous theoretical ${ }^{6}$ and experimental $^{14-16}$ work.

To further investigate this problem we have studied the conductance of several magnetic metals, namely, Fe, Co, and $\mathrm{Ni}$ and a nonmagnetic one, Pt. We have used the mechanically controllable break junction technique that uses a notched wire of the metal under study, which is glued to both sides of the notch on top of a bending beam. By bending of the beam the wire is broken at the notch and with the use of a piezoelement the relative displacement of the two resulting electrodes can be controlled with a precision of a few picometers. The first breaking of the wire is done only at $4.2 \mathrm{~K}$ under cryogenic vacuum in order to assure that the atomic contact is formed between clean electrode surfaces. The break junction device remains under these conditions for the full duration of the experiment. The starting purity of our samples was $99.998 \%$ for $\mathrm{Pt}, \mathrm{Fe}$, and $\mathrm{Ni}$ and of $99.99 \%$ for Co.

As part of the experiments we have recorded traces of conductance while pulling the contacts until breaking (seen as a sudden drop of conductance to well below $1 G_{0}$ ). Once broken the electrodes were pushed together again until the contact was big enough so as to assure a completely new atomic contact configuration. This is important in order to avoid repetitive evolution in the pulling process. ${ }^{17}$ The pro- 

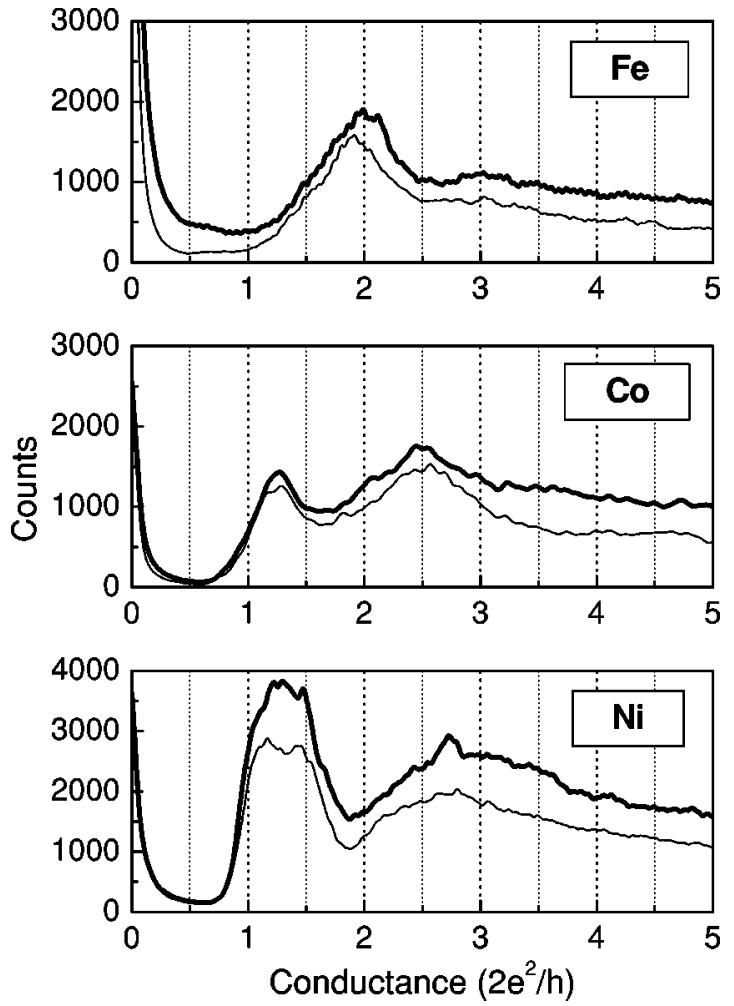

FIG. 1. Conductance histograms for the various metals without magnetic field (thin curve) and when a magnetic field of $5 \mathrm{~T}$ was applied (thick curve). The same structure was observed for every histogram recorded on different samples for the same metals. The conductance was measured using a dc bias voltage of $20 \mathrm{mV}$, and the histograms were constructed using 2500 traces $(\mathrm{Fe}), 1500$ (Co and $\mathrm{Ni}$ at $5 \mathrm{~T}$ ) or 3000 traces $(\mathrm{Ni}$ at $0 \mathrm{~T})$.

cess of contact making followed by controlled breaking was repeated a few thousand times and the traces were used to build histograms of conductance ${ }^{18,19}$ such as those shown in Fig. 1. All traces are included, without any selection.

We collected histograms of conductance for three magnetic metals (Co, Fe, and $\mathrm{Ni}$, Fig. 1) and a nonmagnetic metal, Pt, which has been predicted to become magnetic in a one-dimensional atomic wire configuration. ${ }^{6,20}$ In agreement with previous experiments done under similar conditions, ${ }^{14-16,21-23}$ the histograms for the various $d$ metals in this work show a prominent first peak with a value well above $1 G_{0}$ that is attributed to the conductance of singleatom contacts. Pt is discussed below and looks very similar with a first peak at about $1.5 G_{0}$. Below the first peak the counts rapidly drop until we find a new rise mainly caused by the tunneling of electrons through the vacuum barrier between the two electrodes. Note, in particular, that peaks at $1 G_{0}$ and $\frac{1}{2} G_{0}$ are absent.

To test whether the relative orientation of magnetic domains around the contacts plays any role in our results we have repeated the same experiments in high magnetic fields. Prior to this we measured the hysteresis curves for the sample wires used in the experiments to identify the saturation field, which was for all the cases below $2 \mathrm{~T}$. In the experiments we used fields up to $5 \mathrm{~T}$, well above the saturation fields. The conductance histograms (thick curves in Fig. 1) show no significant difference compared to the zero-field experiments. We even increased the magnetic field up to $10 \mathrm{~T}$ in the case of $\mathrm{Co}$, and again no changes were observed. The small changes observed in Ref. 24 when magnetizing a fixed atomic contact do not appear to survive in an ensemble average. Note that for a given contact magnetostriction can have a significant effect on the contact size and configuration, but by considering conductance histograms our experiment is not affected by such spurious effects.

The present results seem to contradict those obtained in the room-temperature experiments mentioned above. ${ }^{8-13}$ Moreover, if the effects reported for room temperatures were a result of the metals being magnetic these effects should be even be more pronounced at low temperatures. Since this is not the case we have looked for other explanations for the observed (fractional) quantum peaks. The fact that all roomtemperature experiments are performed under atmospheres that are considerably less pure than that provided by cryogenic vacuum we are led to consider the possibility of atomic-scale contamination of the contact by foreign atoms or molecules.

For Pt it has recently been shown that a controlled contamination of the break junction with $\mathrm{H}_{2}$ leads to the appearance of a peak in the conductance histograms situated very close to $1 G_{0}{ }^{23}$ It was shown that this peak in the conductance histogram corresponds to stable configurations of a single hydrogen molecule having a single conductance channel that is nearly perfectly transmitting. Since a hydrogen molecule only allows a single channel of conductance one may guess that when such a molecule bridges two electrodes that are fully spin polarized the conductance would be limited to $\frac{1}{2} G_{0}$. Considering that hydrogen is a rather common contaminant it may have influenced the experiments at room temperature and was acting as a "filter" limiting the number of channels to one.

In order to test this idea we have repeated the experiments in the presence of a hydrogen atmosphere of $\approx 10^{-6}$ mbar. For all the experiments the sample was cooled to $4.2 \mathrm{~K}$ for 1 day in cryogenic vacuum, at this stage we could reproduce the histograms of Fig. 1. Then the $\mathrm{H}_{2}$ gas was introduced and the histograms changed dramatically as shown in Fig. 2. First we note that the histograms show counts at conductances below the first peak (which we will refer to as the "background") in an amount much higher than for the clean metals in Fig. 1. Second, under similar conditions as for the experiment on Pt (Ref. 23) the characteristic peaks for the various magnetic metals are suppressed while a new peak appears near $1 G_{0}$ in the conductance histogram taken at bias voltages above about $100 \mathrm{mV}$. As argued in Ref. 23 the role of the higher bias voltage is to provide some local heating to evaporate away the weakly bound excess hydrogen molecules. The results suggest that the hydrogen-bridged configuration observed for Pt is a general feature in these metals, but we have not yet attempted to confirm this by phonon spectroscopy.

A dominant conductance of $1 G_{0}$ with a hydrogen molecule between electrodes of $\mathrm{Co}, \mathrm{Ni}$, and $\mathrm{Fe}$ suggests that the current is at best partially spin polarized in these atomicsized contacts. As argued in Refs. 5-7, when there is a con- 

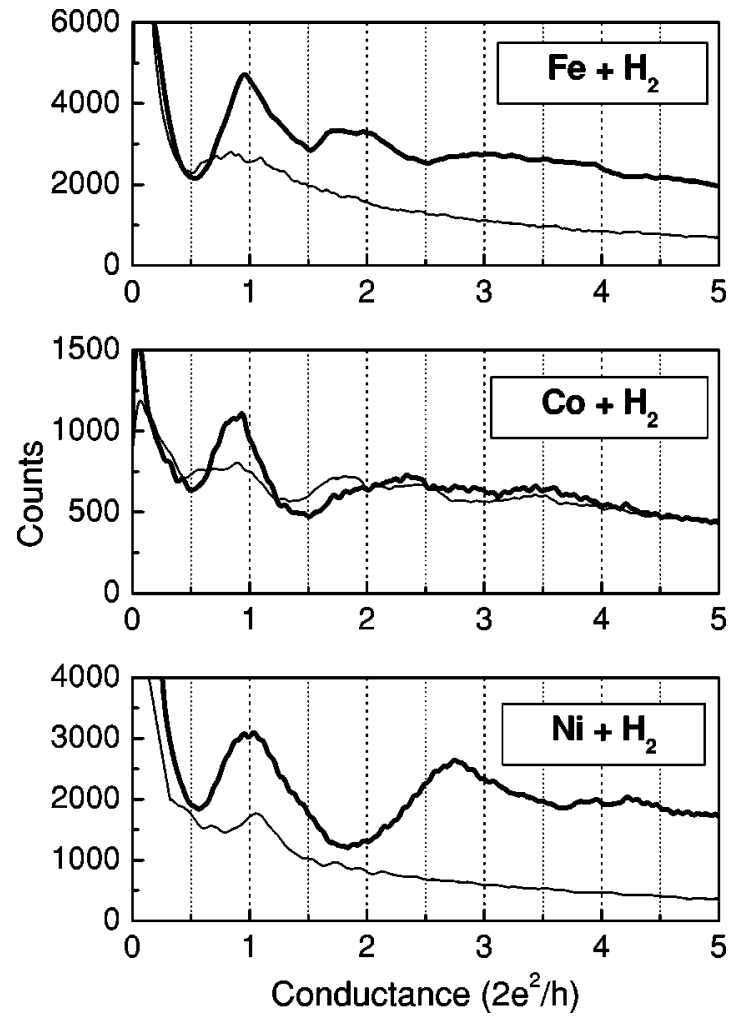

FIG. 2. Conductance histograms for the various magnetic metals in the presence of a hydrogen atmosphere. The traces were recorded using a bias voltage of $20 \mathrm{mV}$ (thin line) and $150 \mathrm{mV}$ (thick line) and the histograms were built from 2000 traces, except for $\mathrm{Ni}$, where we used 1000 (at $20 \mathrm{mV}$ ) and 3000 (at $150 \mathrm{mV}$ ).

tribution of both spin components at the Fermi energy the conductance is still carried by two spin channels even though the spin subbands may be shifted considerably in energy by the exchange interaction. The same is true for Pt. We cannot exclude the possibility of a magnetic moment developing in Pt atomic-sized contacts, as has been predicted. ${ }^{6,20}$ However, its existence cannot be concluded exclusively based on the appearance of peaks at fractional conductance quanta in conductance histograms, as we will illustrate next.

We have searched for effects of other possible gas molecules that could be present in some quantities at room temperatures or in high vacuum. We performed these studies again at low temperatures in a controlled atmosphere. From many experiments we have found that it is easy to tell whether or not some contaminants have reached the sample by just looking at the conductance histograms. In those cases where the contaminants reached the sample there is a large contribution to the histogram for values of conductance below $1 G_{0}$. Above a certain quantity of contaminants the histograms have a large smooth background that decreases with conductance. (Note the difference for the backgrounds between Fig. 1 and the thin lines in Fig. 2). This background is reduced when increasing the applied bias voltage and normally when it is above $200 \mathrm{mV}$ both the new peaks and background disappear and the histograms for the bare metals are recovered.

When introducing small amounts of carbon monoxide, $\mathrm{CO}$, to Pt atomic contacts we observed two new peaks ap-

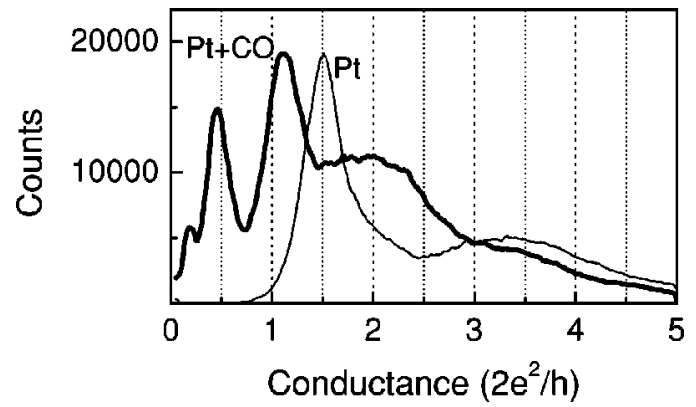

FIG. 3. Conductance histogram for a Pt atomic-sized contact before (thin curve) and after (thick curve) intentional contamination by $\mathrm{CO}$. Although the positions of the peaks at about the first two multiples of $\frac{1}{2} G_{0}$ are suggestive of the lifting of spin degeneracy we argue that the peaks reflect the typical conductance of a $\mathrm{CO}$ molecule between Pt electrodes and are not related to magnetic properties. A bias voltage as high as $150 \mathrm{mV}$ was applied in order to reduce the background.

pearing in the conductance histogram, one near $\frac{1}{2} G_{0}$ and one near $1 G_{0}$ (Fig. 3). The gas was introduced through a capillary heated by sending a current through a resistive wire running inside the full length of the capillary. These peaks behave as those from other contaminants, such as $\mathrm{H}_{2}$, and the positions are remarkably similar to those that would be expected for half-integer quantization. There is, however, no evidence that could relate these peaks to magnetism. The peak near $\frac{1}{2} G_{0}$ likely results from a stable molecular geometry of $\mathrm{CO}$ between the metal electrodes. We would welcome numerical calculations to verify this. Although we cannot claim that the reported observations of half-integer quanta of conductance are all due to contamination of the contacts by $\mathrm{CO}$, our result shows that at least there is one kind of molecule that would produce similar behavior. Since a direct relation of the observed half-integer peaks to magnetism is often not provided, we prefer to tentatively attribute the observed fractional quantum peaks to adsorbed molecular species.

In conclusion, in contrast to previous reports on $\mathrm{Fe}, \mathrm{Co}$, $\mathrm{Ni}$, and Pt we have not detected fractional conductance quantization at low temperatures for the same metals. Our experiments show that the conductance of pure metallic atomicsized contacts for $\mathrm{Fe}, \mathrm{Co}, \mathrm{Ni}$, and $\mathrm{Pt}$ have no significant field dependence in the histogram-averaged conductance, and show no peaks that can be associated with pure quantization, in agreement with recent numerical results. ${ }^{5-7}$ At least part of the previously reported fractional quantization may be explained by the presence of foreign molecules at the surface of the studied samples, as we have demonstrated by intentional contamination of our samples. Our results support the idea that the magnetic state of the samples is not related in a simple manner to its conductance. Although the electrical current in atomic-sized conductors may be partially spin polarized this is not a sufficient condition to obtain a fractional quantum of conductance for single-atom contacts. Only when the conductance is dominated by a single $s$ channel and the exchange energy is large enough to completely block transport through one of the spin subchannels we will find the sought-after half-integer conductance. At the present 
time, the only experiment that probably fulfills these requirements is the one recently reported by Suderow et al., ${ }^{25}$ where the atomic contact is between a gold tip and a thin gold film that is deposited on top of a half-metallic ferromagnetic manganite.
This work is part of the research program of the "Stichting FOM," which is financially supported by NWO. C.U. acknowledges support of the Spanish Ramón y Cajal program of the MCyT. We are grateful to T.G. Sorop for magnetization measurements.
${ }^{1}$ N. Agraï, A. Levy Yeyati, and J. van Ruitenbeek, Phys. Rep. 377, 81 (2003).

${ }^{2}$ E. Scheer, N. Agraï, J. Cuevas, A. Levy Yeyati, B. Ludoph, A. Martín-Rodero, G. Rubio Bollinger, J. van Ruitenbeek, and C. Urbina, Nature (London) 394, 154 (1998).

${ }^{3}$ E. Scheer, P. Joyez, D. Esteve, C. Urbina, and M. Devoret, Phys. Rev. Lett. 78, 3535 (1997).

${ }^{4}$ J. Cuevas, A. Levy Yeyati, A. Martín-Rodero, G. Rubio Bollinger, C. Untiedt, and N. Agrait, Phys. Rev. Lett. 81, 2990 (1998).

${ }^{5}$ A. Martín-Rodero, A. Levy Yeyati, and J. Cuevas, Physica C 352, 67 (2001).

${ }^{6}$ A. Delin and E. Tosatti, Phys. Rev. B 68, 144434 (2003).

${ }^{7}$ A. Bagrets, N. Papanikolaou, and I. Mertig, http://arXiv.org/abs/ cond-mat/0303480.

${ }^{8}$ F. Elhoussine, S. Mátéfi-Tempfli, A. Encinas, and L. Piraux, Appl. Phys. Lett. 81, 1681 (2002).

${ }^{9}$ M. Shimizu, E. Saitoh, H. Miyajima, and Y. Otani, J. Magn. Magn. Mater. 239, 243 (2002).

${ }^{10}$ D. Gillingham, I. Linington, and J. Bland, J. Phys.: Condens. Matter 14, L567 (2002).

${ }^{11}$ D. Gillingham, C. Müller, and J. Bland, J. Phys.: Condens. Matter 15, L291 (2003).

${ }^{12}$ D. Gillingham, I. Linington, C. Müller, and J. Bland, J. Appl. Phys. 93, 7388 (2003).

${ }^{13}$ V. Rodrigues, J. Bettini, P. Silva, and D. Ugarte, Phys. Rev. Lett. 91, 096801 (2003).
${ }^{14}$ C. Sirvent, J. Rodrigo, S. Vieira, L. Jurczyszyn, N. Mingo, and F. Flores, Phys. Rev. B 53, 16086 (1996).

${ }^{15}$ R. Smit, C. Untiedt, A. Yanson, and J. van Ruitenbeek, Phys. Rev. Lett. 87, 266102 (2001).

${ }^{16}$ S. Nielsen, Y. Noat, M. Brandbyge, R. Smit, K. Hansen, L. Chen, A. Yanson, F. Besenbacher, and J. van Ruitenbeek, Phys. Rev. B 67, 245411 (2003).

${ }^{17}$ C. Untiedt, G. Rubio, S. Vieira, and N. Agrait, Phys. Rev. B 56, 2154 (1997).

${ }^{18}$ L. Olesen, E. Lægsgaard, I. Stensgaard, F. Besenbacher, J. Schiøtz, P. Stoltze, K. Jacobsen, and J. Nørskov, Phys. Rev. Lett. 74, 2147 (1995).

${ }^{19}$ J. Krans, J. van Ruitenbeek, V. Fisun, I. Yanson, and L. de Jongh, Nature (London) 375, 767 (1995).

${ }^{20}$ S. Bahn and K. Jacobsen, Phys. Rev. Lett. 87, 266101 (2001).

${ }^{21}$ B. Ludoph and J. van Ruitenbeek, Phys. Rev. B 61, 2273 (2000).

${ }^{22}$ D.J. Bakker, Y. Noat, A.I. Yanson, and J.M. van Ruitenbeek, Phys. Rev. B 65, 235416 (2002).

${ }^{23}$ R. Smit, Y. Noat, C. Untiedt, N. Lang, M. van Hemert, and J. van Ruitenbeek, Nature (London) 419, 906 (2002).

${ }^{24}$ M. Viret, S. Berger, M. Gabureac, F. Ott, D. Olligs, I. Petej, J. Gregg, C. Fermon, G. Francinet, and G.L. Goff, Phys. Rev. B 66, 220401 (2002).

${ }^{25}$ H. Suderow, M. Crespo, S. Vieira, M. Vila, M. Garcia-Hernandez, A. de Andres, C. Prieto, C. Ocal, J. Martinez, and Y. Mukovskii, Physica E (Amsterdam) 18, 264 (2003). 